\title{
ISOTOPY INVARIANTS IN QUASIGROUPS
}

\author{
BY \\ ETTA FALCONER( ${ }^{(1)}$
}

\begin{abstract}
The purpose of this paper is to investigate quasigroup and loop identities that are invariant under isotopy. The varieties of quasigroups and the varieties of loops that are closed under isotopy form isomorphic lattices. Some methods of generating isotopically closed varieties of loops are given.
\end{abstract}

1. Introduction. A major problem in the theory of loops is the isotopyisomorphism problem-When does isotopy imply isomorphism? A closely related, more general problem is the isotopy-invariance problem-What properties are invariant under isotopy? The purpose of this paper is to investigate loop and quasigroup identities that are invariant under isotopy. The associative law and the Moufang identity $(x y \cdot x) z=x(y \cdot x z)$ are well-known identities that are invariant with respect to loop isotopy, [3], [7]. Since varieties are equational classes, an equivalent problem is the determination of all varieties of quasigroups and loops that are closed under isotopy.

$\$ \$ 3$ to 6 are devoted to general considerations of the problem. It is shown that a variety of quasigroups is closed under isotopy if and only if it is the class of all quasigroups all of whose loop isotopes lie in some variety of loops. As a result we obtain a set of quasigroup identities such that every identity invariant under isotopy is either in this set or is equivalent to a member of the set. We next show that the varieties of quasigroups and the varieties of loops that are closed under isotopy form isomorphic lattices, $\mathscr{Q}^{*}$ and $\mathscr{L}^{*}$, respectively, with $\mathscr{L}^{*}$ a sublattice of the lattice of loop varieties. An atom of $2^{*}$ that contains groups has the property that all of its free quasigroups of rank $\boldsymbol{N}_{0}$ or less are isotopic. We also give a variety of loops, which contains no subvariety that is closed under isotopy except the trivial one.

The latter part of the paper is devoted to methods of generating varieties of loops that are isotopically closed. It is shown that the classes of central, left nuclear, right nuclear, and middle nuclear extensions of loops belonging to an isotopically closed loop variety are also isotopically closed varieties. In particular, for each integer $n \geqq 1$, the variety of centrally nilpotent loops of class $m \leqq n$ is isotopically closed.

Received by the editors July 28, 1969.

AMS 1970 Subject Classifications. Primary 20N05; Secondary 06A20, 08A15.

Key Words and Phrases. Variety, isotopically closed variety, inner mapping group, isotope, universal identity, derived identity, anti-associative loop identity.

(1) NSF Science Faculty Fellow. The author is deeply indebted to Dr. Trevor Evans for many valuable suggestions. This study is part of a doctoral dissertation at Emory University.

Copyright (C) 1970, American Mathematical Society 
In addition, we show that any class of loops containing all loop isotopes of its members generates an isotopically closed loop variety. Because of the relationship between isotopically closed quasigroup and loop varieties we can generate an isotopically closed quasigroup variety from any of these new loop varieties by taking all isotopes of the members of the loop variety.

There has been a recent vigorous move in the study of the problem. Evans in [8] showed that a quasigroup is isotopic to a group if and only if it satisfies a law,

$$
\left(\left(x P_{1} \cdot y P_{2}\right) P_{3} \cdot z P_{4}\right) P_{5}=\left(x Q_{1} \cdot\left(y Q_{2} \cdot z Q_{3}\right) Q_{4}\right) Q_{5}
$$

where $P_{i}, Q_{i}, i=1,2,3,4,5$ are permutations on the quasigroup. Belousov in [4] gave identities that are necessary and sufficient conditions that a quasigroup be isotopic to a group or to an abelian group. These identities are invariant under isotopy. Belousov and Ryžkov in [5] stated that there is a one-one correspondence between closure figures in the net determined by a loop and the identities invariant with respect to loop isotopy that are satisfied by the loop. In [12] Osborn studied the structure of loops, all of whose loop isotopes satisfied the identity, $x(x y)^{-1}$ $=y^{-1}$. Aczél in [1] proved that the identities $y(z \cdot y x)=(y \cdot z y) x$ and $(x y \cdot z) y=$ $x(y z \cdot y)$ are invariant with respect to loop isotopy. Other special results have been obtained by Sade and Hosszu, [2], [13].

2. Definitions. A quasigroup is a nonempty set, $Q$, which is closed with respect to three binary operations, multiplication $(\cdot)$, left division $(\mid)$, right division $(/)$, and such that for all $x, y \in Q$,

1. $x \cdot(x \mid y)=y$;

2. $x \mid(x \cdot y)=y$;

3. $(y / x) \cdot x=y$;

4. $(y \cdot x) / x=y$.

A loop is a quasigroup which satisfies $x / x=y \mid y$ for all $x, y$. Here we follow Evans, [11].

A variety of quasigroups is the class of all quasigroups satisfying some given set of quasigroup identities. A variety of loops is likewise defined. Loop varieties, free loops and free quasigroups are discussed in [9], [10], and [11].

A subloop $H$ of a loop $G$ is called a normal subloop of $G$ if for all $x, y \in G$, we have $x H=H x,(H x) y=H(x y)$ and $y(x H)=(y x) H . H$ is said to be fully invariant in $G$, if in addition to being normal, $H \theta \leqq H$ for each endomorphism $\theta$ of $G$. There is a one-one correspondence between the class of loop varieties and the set of fully invariant subloops of the free loop on $\boldsymbol{\aleph}_{0}$ generators [10].

The inner mapping group $I$ of a loop $G$ is the group generated by the mappings $R(x, y)=R_{x} R_{y} R_{x y}^{-1}, L(x, y)=L_{x} L_{y} L_{y x}^{-1}, S(x)=R_{x} L_{x}^{-1}$ for all $x, y \in G$, where $R_{x}$ and $L_{x}$ are the mappings given by $y R_{x}=x y$ and $y L_{x}=y x$, for all $y \in G$. A nonempty subset $S$ of a loop $G$ is called self-conjugate in $G$ if $S I=S$ [6].

The quasigroup $(G, \cdot, \mid, /)$ is said to be isotopic to or an isotope of the quasigroup 
$(H, \circ, \phi, \phi)$ if there exist one-one mappings, $\alpha, \beta, \gamma$ of $G$ upon $H$ such that $x \alpha \circ y \beta$ $=(x \cdot y) \gamma$. If $\gamma$ is the identity mapping on $G$, then $G$ is said to be a principal isotope of $H$. These definitions and basic theorems on isotopy are to be found in Bruck, [6].

3. Isotopically $L$ - and $Q$-closed varieties. A variety $V$ of quasigroups having the property that every isotope of a quasigroup in $V$ also belongs to $V$ is said to be an isotopically $Q$-closed variety. Similarly, an isotopically L-closed variety is a variety of loops which contains all the loop isotopes of its members. The varieties of groups and Moufang loops are isotopically $L$-closed varieties. The variety $V$ of quasigroups satisfying $(x y) / z=(x z) / y$ is an isotopically $Q$-closed variety. By a result of Belousov, [4], any loop in $V$ is an abelian group, clearly of exponent 2. Since groups satisfy the isotopy-isomorphism property, [6], the variety of loops contained in $V$ is isotopically $L$-closed. Moreover, a quasigroup is isotopic to a loop in this variety if and only if all its loop isotopes satisfy $x^{2}=1$, which holds, if and only if the quasigroup satisfies $(x / y) \cdot(z \mid x)=z y$, or equivalently, $(x y) / z$ $=(x z) / y$.

A quasigroup identity is called a universal identity if it is invariant under isotopy. Belousov and Ryžkov have given the name "universal identity" to loop identities having this property with reference to loop isotopy, [5]. It is clear that any identity that is universal under quasigroup isotopy will also be universal under loop isotopy.

Let $(F, \cdot, \mid, /)$ be the free quasigroup on $y, z, x_{1}, x_{2}, \ldots$ and let $(L, \circ, \phi, \phi)$ be the free loop on $x_{1}, x_{2}, \ldots$ If $w$ is a reduced word in $L$, we define its isotopic-related word in $F, \bar{w}$, by

(i) if $w=1$, then $\bar{w}=z y$;

(ii) if $w=x$, then $\bar{w}=x$;

(iii) if $w=u \circ v$, then $\bar{w}=(\bar{u} / y) \cdot(z \mid \bar{v})$;

(iv) if $w=u \phi v$, then $\bar{w}=[\bar{u} /(z \mid \bar{v})] \cdot y$;

(v) if $w=u \phi v$, then $\bar{w}=z \cdot[(\bar{u} / y) \mid \bar{v}]$.

If $w_{1}=w_{2}$ is a loop identity, then $\bar{w}_{1}=\bar{w}_{2}$ is called the derived identity of $w_{1}=w_{2}$.

LEMMA 3.1. Let $(G, \cdot, \mid, /)$ be a quasigroup with principal loop isotope $(G, \circ, \phi, \phi)$ given by $x \circ y=(x / a)(b \mid y)$ for elements $a, b \in G$. Let $g_{1}, g_{2}, \ldots, g_{n}$ be any elements of $G$. If $w\left(x_{1}, x_{2}, \ldots, x_{n}\right)$ is a reduced word in the free loop $(L, \circ, \phi, \phi)$ with isotopicrelated word $\bar{w}\left(x_{1}, x_{2}, \ldots, x_{n}, y, z\right)$ in the free quasigroup $(F, \cdot, 1, /)$, then

$$
w\left(g_{1}, g_{2}, \ldots, g_{n}\right)=\bar{w}\left(g_{1}, g_{2}, \ldots, g_{n}, a, b\right) .
$$

Proof. The proof is by induction on the length of $w$. If $w=1$, then $\bar{w}=z y$. Hence the neutral element of $(G, \circ)$ is $b a=\bar{w}(a, b)$. If $w=x$, then $\bar{w}=x$. Thus $w\left(g_{1}\right)=g_{1}$ $=\bar{w}\left(g_{1}, a, b\right)$. Assume that the assertion holds for words of length less than $k$. Consider $w\left(x_{1}, x_{2}, \ldots, x_{n}\right)$ of length $k>1$. We have three cases: (i) $w=u \circ v$, (ii) $w=u \phi v$, (iii) $w=u \phi v$. By the induction hypothesis

$$
u\left(g_{1}, g_{2}, \ldots, g_{n}\right)=\bar{u}\left(g_{1}, g_{2}, \ldots, g_{n}, a, b\right)
$$


and

$$
v\left(g_{1}, g_{2}, \ldots, g_{n}\right)=v\left(g_{1}, g_{2}, \ldots, g_{n}, a, b\right),
$$

since the length of $u$ and the length of $v$ are both less than the length of $w$. In case (i),

$$
\bar{w}\left(x_{1}, x_{2}, \ldots, x_{n}, y, z\right)=\left[\bar{u}\left(x_{1}, x_{2}, \ldots, x_{n}, y, z\right) / y\right]\left[z \mid \bar{v}\left(x_{1}, x_{2}, \ldots, x_{n}, y, z\right)\right] .
$$

Let $\theta$ be a mapping of the generators of $F$ into $(G, \circ)$ which takes $x_{i}$ into $g_{i}, z$ into $b$, and $y$ into $a$. Since $\theta$ can be extended into a homomorphism of $F$ into $(G, \circ)$, we have

$$
\begin{aligned}
\bar{w}\left(g_{1}, g_{2}, \ldots, g_{n}, a, b\right) & =\left[\bar{u}\left(g_{1}, g_{2}, \ldots, g_{n}, a, b\right) / a\right]\left[b \mid \bar{v}\left(g_{1}, g_{2}, \ldots, g_{n}, a, b\right)\right] \\
& =\left[u\left(g_{1}, g_{2}, \ldots, g_{n}\right) / a\right]\left[b \mid v\left(g_{1}, g_{2}, \ldots, g_{n}\right)\right] \\
& =u\left(g_{1}, g_{2}, \ldots, g_{n}\right) \circ v\left(g_{1}, g_{2}, \ldots, g_{n}\right) \\
& =w\left(g_{1}, g_{2}, \ldots, g_{n}\right) .
\end{aligned}
$$

The proofs of the other cases are analogous to that of the first case.

THEOREM 3.2. $V$ is a variety of quasigroups closed under isotopy if and only if $V$ is the class of all quasigroups, all of whose loop isotopes lie in some variety of loops.

Proof. Let $V$ be an isotopically $Q$-closed variety and let $L$ be the class of loops contained in $V$. $L$ is clearly a loop variety for it is defined by $x / x=y \mid y$ and the identities of $V$. If $G \in L$ and $H$ is a loop isotope of $G$, then $H$ is a quasigroup isotope of a member of $V$ and hence belongs to $V$. But since $H$ is a loop, $H \in L$. Therefore, $L$ is an isotopically $L$-closed variety.

For the converse, we assume that $V$ is the class of all quasigroups, all of whose loop isotopes lie in the variety of loops, $L$. Let $I$ be a defining set of identities for $L$ and let $I^{\prime}$ be the set of derived identities of $I$. Then $I^{\prime}$ defines a variety of quasigroups $Q\left(I^{\prime}\right)$. We will show that $V=Q\left(I^{\prime}\right)$.

Let $G$ be a quasigroup in $Q\left(I^{\prime}\right)$ with principal loop isotope $H$ given by $x \circ y$ $=(x / a)(b \backslash y)$ for some elements $a, b \in G$. Let $w_{1}\left(x_{1}, x_{2}, \ldots, x_{n}\right)=w_{2}\left(x_{1}, x_{2}, \ldots, x_{n}\right)$ be an identity in $I$ and $g_{1}, g_{2}, \ldots, g_{n} \in G$. $G$ satisfies $\bar{w}_{1}=\bar{w}_{2}$. Hence

$$
\bar{w}_{1}\left(g_{1}, g_{2}, \ldots, g_{n}, a, b\right)=\bar{w}_{2}\left(g_{1}, g_{2}, \ldots, g_{n}, a, b\right)
$$

and so, $H$ is a member of $L$. Now, any loop isotope of $G$ is isomorphic to a principal loop isotope of $G$ and since varieties are closed under homomorphic images, it follows that every loop isotope of $G$ belongs to $L$. But this means that $G$ is in $V$. Hence $Q\left(I^{\prime}\right) \subseteq V$.

Next, we suppose that $G \in V$ and that

$$
\bar{w}_{1}\left(x_{1}, x_{2}, \ldots, x_{n}, y, z\right)=\bar{w}_{2}\left(x_{1}, x_{2}, \ldots, x_{n}, y, z\right)
$$

is an identity in $I^{\prime}$. Let $g_{1}, g_{2}, \ldots, g_{n}, a, b \in G$ and let $H$ be the principal loop isotope of $G$ given by $x \circ y=(x / a)(b \mid y)$. Since $H \in L, H$ satisfies $w_{1}=w_{2}$ and so, 
$w_{1}\left(g_{1}, g_{2}, \ldots, g_{n}\right)=w_{2}\left(g_{1}, g_{2}, \ldots, g_{n}\right)$. It follows from Lemma 3.1 that

$$
\bar{w}_{1}\left(g_{1}, g_{2}, \ldots, g_{n}, a, b\right)=\bar{w}_{2}\left(g_{1}, g_{2}, \ldots, g_{n}, a, b\right) \text {. }
$$

Since $G$ satisfies the defining identities of $Q\left(I^{\prime}\right), G \in Q\left(I^{\prime}\right)$ and so $V \subseteq Q\left(I^{\prime}\right)$. Thus, $V$ is a variety.

It remains to show that $V$ is isotopically $Q$-closed. Let $G \in V$ and let $H$ be any isotope of $G$. Then, any loop isotope of $H$ is a loop isotope of $G$ and hence, belongs to $L$. Since every loop isotope of $H$ is in $L, H \in V$. Therefore, $V$ is isotopically $Q$-closed.

COROllary 3.3. A derived identity is a universal identity.

Proof. By Theorem 3.2 and its proof, the variety $V$ defined by $\bar{w}_{1}=\bar{w}_{2}$ is isotopically $Q$-closed. But then, if $Q$ is any quasigroup satisfying $\bar{w}_{1}=\bar{w}_{2}$, so is every isotope of $Q$. Therefore, $\bar{w}_{1}=\bar{w}_{2}$ is a universal identity.

4. The lattices of isotopically $L$ - and $Q$-closed varieties. We now introduce the following notation. If $L$ is a loop variety, then $Q^{*}(L)$ will denote the variety of quasigroups, all of whose loop isotopes lie in $L$, and $L^{*}$, the variety of loops contained in $Q^{*}(L)$. We have seen in the preceding section that $Q^{*}(L)$ is the variety defined by the derived identities of any defining set of identities for $L$ and that both $Q^{*}(L)$ and $L^{*}$ are closed under isotopy.

THEOREM 4.1. $L^{*}$ is the maximum isotopically $L$-closed subvariety of $L$.

Proof. $L^{*}$ is clearly a subvariety of $L$, for if $G \in L^{*} \subseteq Q^{*}(L)$, then every loop isotope of $G$ is in $L$. But since isotopy is an equivalence relation, this means that $G$, itself, is in $L$. Now, if $L^{\prime}$ is any isotopically $L$-closed subvariety of $L$ and $G$ is any loop in $L^{\prime}$, then every loop isotope of $G$ is in $L^{\prime}$ and hence, in $L$. Therefore $G$ is a loop in $Q^{*}(L)$ and hence, belongs to $L^{*}$.

THEOREM 4.2. $\mathscr{L}^{*}$, the class of all isotopically L-closed varieties, is a sublattice of the lattice of loop varieties.

Proof. Let $U$ and $\cap$ denote the join and meet operations in the lattices of loop and quasigroup varieties. We need only show that the join and meet of two isotopically $L$-closed varieties $L_{1}^{*}$ and $L_{2}^{*}$ are also isotopically $L$-closed varieties. This is trivial for the meet, $L_{1}^{*} \cap L_{2}^{*}$, for if $G \in L_{1}^{*} \cap L_{2}^{*}$, then every loop isotope of $G$ belongs to $L_{1}^{*}$ and to $L_{2}^{*}$, and hence to their meet.

To show that the same is true of the join, we first note that if $L^{*} \subseteq L_{a}^{*}$, then $Q^{*}\left(L^{*}\right) \subseteq Q^{*}\left(L_{a}^{*}\right)$. This holds since a quasigroup in $Q^{*}\left(L^{*}\right)$ must have all its loop isotopes in $L^{*}$ and hence, in $L_{a}^{*}$. But $Q^{*}\left(L_{a}^{*}\right)$ is the variety of all quasigroups all of whose loop isotopes lie in $L_{a}^{*}$. Therefore $Q^{*}\left(L^{*}\right) \subseteq Q^{*}\left(L_{a}^{*}\right)$. In view of this fact, we have $L_{1}^{*} \cup L_{2}^{*} \subseteq Q^{*}\left(L_{1}^{*}\right) \cup Q^{*}\left(L_{2}^{*}\right) \subseteq Q^{*}\left(L_{1}^{*} \cup L_{2}^{*}\right)$. But the variety of loops in $Q^{*}\left(L_{1}^{*} \cup L_{2}^{*}\right)$ is $\left(L_{1}^{*} \cup L_{2}^{*}\right)^{*}$. Hence $L_{1}^{*} \cup L_{2}^{*} \subseteq\left(L_{1}^{*} \cup L_{2}^{*}\right)^{*}$. By Theorem 4.1 $\left(L_{1}^{*} \cup L_{2}^{*}\right)^{*} \subseteq L_{1}^{*} \cup L_{2}^{*}$ and hence, equality follows. Since $\left(L_{1}^{*} \cup L_{2}^{*}\right)^{*}$ is isotopically $L$-closed, so is $L_{1}^{*} \cup L_{2}^{*}$. 
THEOREM 4.3. $2^{*}$, the class of isotopically $Q$-closed varieties, is a lattice, isomorphic to $\mathscr{L}^{*}$.

Proof. $\mathscr{Q}^{*}$ is clearly ordered under $\subseteq$. The isomorphism between $\mathscr{Q}^{*}$ and $\mathscr{L}^{*}$ is the obvious one, $L^{*} \stackrel{\theta}{\rightarrow} Q^{*}\left(L^{*}\right) . \theta$ is order preserving for $L_{1}^{*} \subseteq L_{2}^{*}$ if and only if $Q^{*}\left(L_{1}^{*}\right) \subseteq Q^{*}\left(L_{2}^{*}\right)$. Moreover, since $Q^{*}\left(L^{*}\right)$ consists of all quasigroups isotopic to loops in $L^{*}$, we have $L_{1}^{*} \subset L_{2}^{*}$ if and only if $Q^{*}\left(L_{1}^{*}\right) \subset Q^{*}\left(L_{2}^{*}\right)$. Therefore $\theta$ is a lattice isomorphism between $\mathscr{2}^{*}$ and $\mathscr{L}^{*}$.

The variety $V_{L}$ of all loops and the variety $V_{Q}$ of all quasigroups are the greatest elements in $\mathscr{L}^{*}$ and $\mathscr{2}^{*}$, respectively. The least element in both lattices is $E$, the trivial variety. Since every group variety belongs to $\mathscr{L}^{*}, \mathscr{Q}^{*}$ contains a sublattice which is isomorphic to the lattice of group varieties.

Although $2^{*}$ is a lattice isomorphic to a sublattice of loop varieties, we have been unable to establish that $2^{*}$ is a sublattice of the lattice of quasigroup varieties. One difficulty lies in the fact that even though our defining set of identities of a variety in $2^{*}$ consists only of universal identities, it is not the case that every identity implied by these identities and hence holding in all members of the variety, is a universal identity. The universal identity $(x / y)(z \mid x)=z y$ implies the identity $x\left(x^{2} \mid x^{2}\right)=x^{2} \cdot x$ which is satisfied by the loop $G$ below, but not by its isotope $H$.

\begin{tabular}{l|l|l|l|l|l}
$G$ & 1 & 2 & 3 & 4 & 5 \\
\hline 1 & 1 & 2 & 3 & 4 & 5 \\
\hline 2 & 2 & 1 & 5 & 3 & 4 \\
\hline 3 & 3 & 4 & 1 & 5 & $\frac{2}{2}$ \\
\hline 4 & 4 & 5 & 2 & 1 & 3 \\
\hline 5 & 5 & 3 & 4 & 2 & 1
\end{tabular}

\begin{tabular}{l|l|l|l|l|l}
$H$ & 1 & 2 & 3 & 4 & 5 \\
\hline 1 & 4 & 5 & 2 & 1 & 3 \\
\hline 2 & 5 & 3 & 4 & 2 & 1 \\
\hline 3 & 1 & 2 & 3 & 4 & 5 \\
\hline 4 & 3 & 4 & 1 & 5 & $\frac{2}{4}$ \\
\hline 5 & 2 & 1 & 5 & 3 & 4
\end{tabular}

$G$ is a loop satisfying $x^{2}=1$ and hence, clearly satisfies the identity given above. In $H$, the element 1 fails to satisfy the identity. Therefore, the identity is not invariant under isotopy.

Another difficulty lies in the fact that if $Q$ and $L$ are quasigroup and loop varieties such that $L^{*} \subset Q \subseteq Q^{*}(L)$, then it is not necessarily the case that $Q=Q^{*}(L)$. The quasigroup isotope $K$ of $C_{2} \times C_{2}$

\begin{tabular}{l|l|l|l|l}
$K$ & 1 & 2 & 3 & 4 \\
\hline 1 & 1 & 3 & 4 & 2 \\
\hline 2 & 3 & 1 & 2 & 4 \\
\hline 3 & 4 & 2 & 1 & 3 \\
\hline 4 & 2 & 4 & 3 & 1
\end{tabular}


satisfies $x^{2}=y^{2}, x y=y x$ and belongs to the variety $Q^{*}\left(A_{2}\right)$ where $A_{2}$ is the variety of abelian groups satisfying $x^{2}=1$. Therefore, the subvariety, $V$ of $Q^{*}\left(A_{2}\right)$ determined by $x^{2}=y^{2}, x y=y x$ is not $A_{2}$, since it contains $K$. We have $A_{2} \subset V \subseteq Q^{*}\left(A_{2}\right)$ but $V \neq Q^{*}\left(A_{2}\right)$.

THEOREM 4.4. A quasigroup identity is invariant under isotopy if and only if it is equivalent to a derived identity.

Proof. Let $w_{1}=w_{2}$ be a quasigroup identity that is equivalent to a derived identity, $\bar{w}_{3}=\bar{w}_{4}$. By Corollary $3.3, \bar{w}_{3}=\bar{w}_{4}$ is a universal identity. But a quasigroup satisfies $w_{1}=w_{2}$ if and only if it satisfies $\bar{w}_{3}=\bar{w}_{4}$. Hence, $w_{1}=w_{2}$ is invariant under isotopy.

On the other hand, if $w_{1}=w_{2}$ is invariant under isotopy and is not a derived identity, let $V_{1}$ and $V_{2}$ be the quasigroup varieties defined by $w_{1}=w_{2}$ and $\bar{w}_{1}=\bar{w}_{2}$, respectively. These varieties are closed under isotopy with respect to their defining identities. If $G$ is a loop in $V_{1}$, then $G$ satisfies $\bar{w}_{1}=\bar{w}_{2}$ since all its loop isotopes lie in $V_{1}$. If $G$ is a loop in $V_{2}$, then $G$ satisfies

$$
\bar{w}_{1}\left(x_{1}, x_{2}, \ldots, x_{n}, y, z\right)=\bar{w}_{2}\left(x_{1}, x_{2}, \ldots, x_{n}, y, z\right) .
$$

Setting $y=z=1$, we see that $G$ satisfies

$$
\bar{w}_{1}\left(x_{1}, x_{2}, \ldots, x_{n}, 1,1\right)=\bar{w}_{2}\left(x_{1}, x_{2}, \ldots, x_{n}, 1,1\right) .
$$

A simple induction argument shows that this is the identity $w_{1}=w_{2}$. Thus, if $L_{1}$ and $L_{2}$ are the varieties of loops in $V_{1}$ and $V_{2}$, then $L_{1}=L_{2}$. But by Theorem 3.2, $L_{1}$ and $L_{2}$ are isotopically $L$-closed varieties. Therefore, it follows from Theorem 4.3 that $V_{1}=V_{2}$ which establishes the equivalence of $w_{1}=w_{2}$ and its derived identity.

In view of the preceding theorem, the study of universal identities reduces to the study of derived identities. In fact, if $S$ is the set of all loop identities $w_{1}=w_{2}$ on $x_{1}, x_{2}, \ldots$ with $w_{1}$ and $w_{2}$ reduced words, then Theorem 4.4 implies that the set of derived identities of $S, S^{\prime}$, has the property that every universal identity is either in $S^{\prime}$ or is equivalent to a member of $S^{\prime}$. It can also be shown that any loop identity that is universal for loop isotopy is either a derived identity or equivalent to its derived identity. Thus, for example, the Moufang identity, $(x y \cdot z) y=x(y \cdot z y)$ is equivalent to its derived identity,

$$
([([(x / u)(v \mid y)] / u)(v \mid z)] / u)(v \mid y)=(x / u)(v \backslash[(y / u)(v \mid[(z / u)(v \mid y)])]) .
$$

5. The atoms $Q^{*}\left(A_{p}\right)$. The lattice $\mathscr{G}$ of group varieties is a sublattice of the lattice of isotopically $L$-closed varieties. By Theorem 4.3 , the sublattice $\mathscr{S}$ of isotopically $Q$-closed varieties consisting of all varieties $Q^{*}(L)$ where $L$ is a group variety is isomorphic to $\mathscr{G}$. Since the atoms of $\mathscr{G}$ are known to be the varieties $A_{p}$ defined by $x \cdot y z=x y \cdot z, x y=y x, x^{p}=1$, for $p$, a prime, the atoms of $\mathscr{S}$ will be the varieties of quasigroups $Q^{*}\left(A_{p}\right)$ defined by

(i) $x(v \mid[(y / u) z])=([x(v \mid y)] / u) z$; 
(ii) $x[z \mid(y u)]=y[z \mid(x u)]$;

(iii) $((\cdots)(((y x) / z) x) z) \cdots x) / z) x=y z$ where $x$ occurs $p$ times.

For each cardinal $n$, there is at most one group within isomorphism of order $n$ in $A_{p}$. In other words, all groups in $A_{p}$ with cardinality $n$ are isomorphic. Every quasigroup in $Q^{*}\left(A_{p}\right)$ with cardinality $n$ must be isotopic to a group in $A_{p}$ of the same cardinality. Therefore we have the following result.

Lemma 5.1. All quasigroups in $Q^{*}\left(A_{p}\right)$ of cardinality $n$ are isotopic.

The preceding lemma implies that free quasigroups in $Q^{*}\left(A_{p}\right)$ are isotopic if they have the same cardinality. It is a well-known fact that two free quasigroups in a variety on $\alpha$ and $\beta$ generators where $\alpha$ and $\beta$ are different infinite cardinals cannot be isotopic since the cardinality of the free quasigroups will be $\alpha$ and $\beta$ respectively. However, it is possible for two free quasigroups in a variety to have the same cardinality if each has a finite or countably infinite number of free generators.

THEOREM 5.2. All free quasigroups in $Q^{*}\left(A_{p}\right)$ on a countably infinite or finite number of free generators are isotopic.

Proof. We will show that the free quasigroup on one generator in $Q^{*}\left(A_{p}\right)$ is infinite. Let $C_{p}$ be the group in $A_{p}$ of order $p$ and let $C$ be the restricted direct product of $\aleph_{0}$ copies of $C_{p}$, where we take the generators of the copies of $C_{p}$ to be $a_{1}, a_{2}, a_{3}, \ldots$ Let $\alpha, \beta$ be permutations on $C$ given by

(i) for $x \in C, x \neq a_{i}, x \alpha=x \beta=x, a_{1} \beta=a_{1}, a_{2} \beta=a_{2}, a_{3} \beta=a_{4}$;

(ii) for $n \geqq 0, a_{3 n+1} \alpha=a_{3 n+2}, a_{3 n+2} \alpha=a_{3 n+3}, a_{3 n+3} \alpha=a_{3 n+1}$;

(iii) for $n \geqq 1, a_{3 n+1} \beta=a_{3 n+2}, a_{3 n+2} \beta=a_{3 n}, a_{3 n+3} \beta=a_{3 n+4}$.

Let $G$ be the isotope of $C$ given by $x \circ y=x \alpha \cdot y \beta$ and let $B$ be the subquasigroup of $G$ generated by $a_{1}$. First we note that $a_{1} \circ a_{1}=a_{2} a_{1} ; a_{2} a_{1} \circ a_{2} a_{1} \circ \cdots \circ a_{2} a_{1}=1$ ( $p$ times); $1 \circ a_{1}=a_{1} ; a_{1} \circ 1=a_{2} ; a_{2} \circ 1=a_{3} ; 1 \circ a_{3}=a_{4}$. Therefore for $n=0, a_{3 n+1}$, $a_{3 n+2}, a_{3 n+3}$ are generated by $a_{1}$. Assume that for $n \leqq k$, that this same triple is generated by $a_{1}$. Then

$1 \circ a_{3 k+3}=a_{3 k+4}=a_{3(k+1)+1} ; a_{3(k+1)} \circ 1=a_{3(k+1)+2} ; a_{3(k+1)+2} \circ 1=a_{3(k+1)+3}$.

Therefore, for each nonnegative integer $i, a_{i} \in B$. Since $B$ is an infinite one-generator subquasigroup and belongs to $Q^{*}\left(A_{p}\right)$, the free quasigroup on one generator in this variety is infinite. Therefore every free quasigroup in $Q^{*}\left(A_{p}\right)$ with $\boldsymbol{\aleph}_{0}$ or fewer generators has cardinality $\aleph_{0}$. By Lemma 5.1, these quasigroups are isotopic.

COROLLARY 5.3. Every free quasigroup in an isotopically $Q$-closed variety containing groups is infinite.

6. Anti-associative loop varieties. Every variety of loops, $L$, determines an isotopically $L$-closed variety $L^{*}$. However, $L^{*}$ may be the trivial loop variety in which case $Q^{*}(L)$ is also trivial. $L^{*}$ is nontrivial if and only if the derived identities 
defining $Q^{*}(L)$ are consistent. T. Evans in [9] defines an anti-associative loop variety to be a variety of loops containing no nontrivial groups and shows that every anti-associative loop variety has as one of its defining identities, an antiassociative loop identity, that is, an identity which is incompatible with associativity in loops. It is easy to see that if $L$ is not anti-associative, then $L^{*}$ is nontrivial, for in this case $L$ must contain a nontrivial variety of groups. Since every variety of groups is an isotopically $L$-closed variety, and $L^{*}$ is the maximum subvariety of $L$ that is closed under isotopy, $L^{*}$ cannot be the trivial variety.

We have been unable to prove the existence of an anti-associative isotopically $L$-closed variety that is nontrivial. However, the following example does establish the existence of a variety of loops containing no isotopically $L$-closed subvariety except the trivial one.

Let $(G, \cdot)$ be the nine element loop satisfying $x[x(1 / x)]=1$ with isotope $(H, \circ)$ given by $x \circ y=(x / 2)(8 \mid y)$.

\begin{tabular}{c|c|c|c|c|c|c|c|c|c}
$G$ & 1 & 2 & 3 & 4 & 5 & 6 & 7 & 8 & 9 \\
\hline 1 & 1 & 2 & 3 & 4 & 5 & 6 & 7 & 8 & 9 \\
\hline 2 & 2 & 6 & 1 & 3 & 8 & 5 & 9 & 4 & 7 \\
\hline 3 & 3 & 5 & 8 & 6 & 1 & 7 & 4 & 9 & 2 \\
\hline 4 & 4 & 1 & 9 & 7 & 2 & 8 & 6 & 5 & 3 \\
\hline 5 & 5 & 7 & 4 & 1 & 9 & 3 & 2 & 6 & 8 \\
\hline 6 & 6 & 9 & 5 & 8 & 3 & 2 & 1 & 7 & 4 \\
\hline 7 & 7 & 8 & 6 & 5 & 4 & 9 & 3 & 2 & 1 \\
\hline 8 & 8 & 4 & 2 & 9 & 7 & 1 & 5 & 3 & 6 \\
\hline 9 & 9 & 3 & 7 & 2 & 6 & 4 & 8 & 1 & 5
\end{tabular}

\begin{tabular}{|c|c|c|c|c|c|c|c|c|c|}
\hline & 1 & 2 & 3 & 4 & 5 & 6 & 7 & 8 & \\
\hline 1 & 8 & 9 & 5 & 1 & 6 & 3 & 2 & 4 & 7 \\
\hline 2 & 6 & 3 & 8 & 2 & 7 & 9 & 5 & 1 & 4 \\
\hline 3 & 4 & 7 & 1 & 3 & 8 & 5 & 6 & 9 & 2 \\
\hline 4 & 1 & 2 & 3 & 4 & 5 & 6 & 7 & 8 & 9 \\
\hline 5 & 7 & 8 & 9 & 5 & 4 & 2 & 1 & 3 & 6 \\
\hline 6 & 5 & 1 & 4 & 6 & 9 & 7 & 8 & 2 & 3 \\
\hline 7 & 3 & 4 & 6 & 7 & 2 & 8 & 9 & 5 & 1 \\
\hline 8 & 9 & 6 & 2 & 8 & 3 & 1 & 4 & 7 & 5 \\
\hline 9 & 2 & 5 & 7 & 9 & 1 & 4 & 3 & 6 & 8 \\
\hline
\end{tabular}

$G$ is anti-associative, since no group except the trivial one can satisfy the identity $x[x(1 / x)]=1$, but $H$ is not, since $H$ contains the subgroup $\{4,5\}$. Therefore the variety $L$ generated by $G$ is not closed under isotopy.

We will construct the polynomial loop $F$ on one generator in the variety $L$. Using functional notation, $F$ is generated by the function $f \in G^{9}$ defined by $f(x)=x$. We will use the notation $x^{n}$ and ${ }^{n} x$ which are defined by

(i) $x^{1}={ }^{1} x=x$;

(ii) $x^{n+1}=x^{n} \cdot x ;{ }^{n+1} x=x \cdot{ }^{n} x$.

With some computation we can find the following elements in $F$ :

$$
\begin{aligned}
& f_{9}={ }^{210} f=(1,1,1,1,1,1,1,1,6), \\
& f_{7}={ }^{126} f=(1,1,1,1,1,1,7,1,1), \\
& f_{3}={ }^{315} f=(1,1,9,1,1,1,1,1,1),
\end{aligned}
$$




$$
\begin{aligned}
f_{5} & =\left(f_{7}^{4}\right) f^{36}=(1,1,1,1,5,1,1,1,1), \\
g & =\left(f_{5}^{4}\left(f_{7}^{4}\left(f \cdot{ }^{5} f_{9}\right)\right)\right) f_{3}^{2}=(1,2,1,4,1,6,1,8,1), \\
f_{8} & =\left({ }^{4} g \cdot g^{2}\right)^{5}=(1,1,1,1,1,1,1,4,1), \\
f_{4} & =\left(g \cdot{ }^{3} f_{8}\right)^{9}=(1,1,1,4,1,1,1,1,1), \\
f_{2} & =\left(\left(f_{4}^{3}\left(g \cdot{ }^{3} f_{8}\right)\right)^{3}\right)^{5}=(1,4,1,1,1,1,1,1,1), \\
f_{6} & =f_{2}^{2} \cdot\left(f_{4}^{3}\left(g \cdot{ }^{3} f_{8}\right)\right)^{3}=(1,1,1,1,1,5,1,1,1) .
\end{aligned}
$$

Since $G$ is generated by each of its elements different from $1, f_{i}, i=2,3, \ldots, 9$ generates an isomorphic copy of $G$. Therefore $F=G_{1} \times G_{2} \times \cdots \times G_{8}$ where $G_{i} \cong G, i=1,2, \ldots, 8$.

Now, suppose that $L$ contains a nontrivial subvariety $L^{\prime}$ that is isotopically $L$-closed. Let $F^{\prime}$ be the free loop in $L^{\prime}$ on one generator. Since $F$ was constructed to be the free loop in $L$ on one generator, there is a homomorphism $\theta$ of $F$ onto $F^{\prime}$. Since $F^{\prime}=F \theta=\left(G_{1} \times G_{2} \times \cdots \times G_{8}\right) \theta, F^{\prime}$ is generated by $G_{1} \theta, G_{2} \theta, \ldots, G_{8} \theta$. It is easily verified that the only homomorphic images of $G$ are 1 and $G$ itself. Thus, for $i=1,2, \ldots, 8, G_{i} \theta=1$ or $G_{i}$. Let $x_{i}$ be the element of $F$ whose $i$ th component is $x$ and whose other components are 1 . If $F^{\prime} \neq 1$, then there exists an integer $i, 1 \leqq i \leqq 8$ such that for all $x, y \in G_{i}, x \theta \neq y \theta$, for if not, then $G_{i} \theta=1, i=1,2, \ldots, 8$, from which it follows that $F^{\prime}=1$. Consider the isotope of $F, K$, given by $x \circ y=\left(x / 2_{i}\right)\left(8_{i} \mid y\right)$. The identity element of $K$ is $4_{i}$ and $5_{i} \circ 5_{i}=\left(5_{i} / 2_{i}\right)\left(8_{i} \mid 5_{i}\right)=4_{i}$. Hence $K$ contains the nontrivial subgroup $\left\{4_{i}, 5_{i}\right\}$. Now $\theta$ induces a homomorphism $\theta$ o of $K$ onto $K^{\prime}$ where $K^{\prime}$ is an isotope of $F^{\prime}$ and $x \theta \circ=x \theta$. Since $4_{i} \theta \neq 5_{i} \theta, 4_{i} \theta \circ \neq 5_{i} \theta \circ$. Therefore $\left\{4_{i} \theta \circ, 5_{i} \theta \circ\right\}$ is a two element subgroup of $K^{\prime}$. Since $K^{\prime}$ is not anti-associative, the variety $L^{\prime}$ is not isotopically $L$-closed. Therefore, $L$ contains no isotopically $L$-closed subvariety except the trivial one.

7. Some special examples of isotopically $L$-closed varieties. If we are given two isotopically $L$-closed varieties, neither containing the other, then we can find two new isotopically $L$-closed varieties by taking the join and meet of the two varieties. In this section we will give a method of constructing new isotopically $L$-closed varieties from a single isotopically $L$-closed variety.

If $x, y$ and $z$ are elements of a loop $G$, then the commutator of $x$ and $y$ is $[x, y]$ $=x y / y x$. The associator of $x, y$, and $z$ is $[x, y, z]=(x y \cdot z) /(x \cdot y z)$. The left nucleus of $G$ is the set of all $a \in G$ such that $(a x) y=a(x y)$ for all $x, y \in G$. The middle nucleus of $G$ is similarly defined in terms of $(x a) y=x(a y)$, and the right nucleus in terms of $(x y) a=x(y a)$. The semicenter is the subloop generated by all $a \in G$ such that $a x=x a$ for all $x \in G$. The center $Z(G)$ is the set of all elements in the intersection of the three nuclei that commute with every element of $G$. The three nuclei are subgroups of $G$ and the center is an abelian subgroup, [6].

Let $F$ be the free loop on a countably infinite number of generators, $x_{1}, x_{2}, \ldots$ Let $I$ be the inner mapping group of $F$ and $N$, a fully invariant subloop of $F$. We 
consider the following subloops of $F$.

$$
\begin{aligned}
& U_{1}=\langle[n, u] \alpha \mid n \in N, u \in F, \alpha \in I\rangle, \\
& U_{2}=\langle[n, u, v] \alpha \mid n \in N, y, v \in F, \alpha \in I\rangle, \\
& U_{3}=\langle[u, n, v] \alpha \mid n \in N, u, v \in F, \alpha \in I\rangle, \\
& U_{4}=\langle[u, v, n] \alpha \mid n \in N, u, v \in F, \alpha \in I\rangle, \\
& U_{5}=\left\langle U_{1}, U_{2}, U_{3}, U_{4}\right\rangle .
\end{aligned}
$$

LEMMA 7.1. $U_{1}-U_{5}$ are fully invariant subloops of $F$.

Proof. The first four subloops are normal in $F$, since they are generated by selfconjugate subsets, [6, p. 63]. Let $\theta$ be an endomorphism of $F$. Since $N$ is fully invariant, if $[n, u] \alpha$ is a generator of $U_{1}$, then $([n, u] \alpha) \theta=[n \theta, u \theta] \alpha^{\prime} \in U_{1}$, where $\alpha^{\prime} \in I$ is obtained by replacing every $x_{i}$ involved in $\alpha$ by $x_{i} \theta$. Because $U_{1} \theta$ is generated by the images of the generators of $U_{1}$, we have $U_{1} \theta \leqq U_{1}$.

A similar argument shows that $U_{2}, U_{3}$, and $U_{4}$ are also fully invariant. As a subloop generated by four fully invariant subloops, $U_{5}$ is fully invariant.

We will denote the varieties determined by the fully invariant subloops, $U_{1}, U_{2}$, $U_{3}, U_{4}, U_{5}, N$ by $L_{1}, L_{2}, L_{3}, L_{4}, L_{5}$, and $L$, respectively. We note that any identity satisfied in one of the varieties is loop equivalent to an identity $w=1$, where $w$ is an element of the corresponding fully invariant subloop of $F$. The elements of the first four subloops are easily seen to be words in the generators. For example, an identity in $L_{1}$ is equivalent to an identity of the form,

$$
\begin{aligned}
& w\left(\left[n_{1}(x), w_{1}(x)\right] \alpha_{1} \cdots\left[n_{t}(x), w_{t}(x)\right] \alpha_{t}\right)=1, \\
& \quad \text { where } \alpha_{i} \in I, n_{i} \in N, w_{i} \in F, i=1,2, \ldots, t,
\end{aligned}
$$

and where we are abbreviating a word $w\left(x_{1}, x_{2}, \ldots, x_{n}\right)$ by $w(x)$. Since $U_{5}$ is generated by normal subloops, $U_{5}=U_{1} U_{2} U_{3} U_{4}$. Thus, any identity satisfied in $L_{5}$ is equivalent to an identity of the form $\left(w_{1} w_{2} \cdot w_{3}\right) w_{4}=1$ where $w_{i}$ is a word in $U_{i}$. It is clear in view of the fact that the lattice of loop varieties is isomorphic to the lattice of fully invariant subloops of $F$, that $L_{5}$ is the intersection of the varieties $L_{1}, L_{2}, L_{3}$, and $L_{4}$. Morcover, $L \subseteq L_{5}$, since $U_{5} \subseteq N$. It is known that in certain cases, $L_{2}, L_{3}, L_{4}$, and $L_{5}$ are one and the same variety, for example, if $L$ is the variety of Moufang loops.

THEOREM 7.2. The varieties, $L_{1}-L_{5}$, are the classes $C_{1}-C_{5}$ of all loops containing a normal subloop in the semicenter, left nucleus, middle nucleus, right nucleus or center, respectively, with quotient loop in L.

Proof. We will show first that the free loops in $L_{1}$ are in $C_{1}$. Let $n$ be any cardinal. Since $N$ and $U_{1}$ are fully invariant subloops of $F$, the free loops on $n$ generators $s_{1}, s_{2}, \ldots$ in $L_{1}$ and $L$ are given by $F_{n} / U_{1}\left(F_{n}\right)$ and $F_{n} / N\left(F_{n}\right)$, respectively. Since $U_{1}\left(F_{n}\right) \triangleleft N\left(F_{n}\right)$, there is a homomorphism $\theta$ from $F_{n} / U_{1}\left(F_{n}\right)$ onto $F_{n} / N\left(F_{n}\right)$ defined by $w U_{1}\left(F_{n}\right) \theta=w N\left(F_{n}\right)$ where $w \in F_{n}$. The kernel of $\theta$ is $N\left(F_{n}\right) / U_{1}\left(F_{n}\right)$. If $a \in \operatorname{ker} \theta$ and $b \in F_{n} / U_{1}\left(F_{n}\right)$, then $[a, b]=[n(s), w(s)] U_{1}\left(F_{n}\right)=U_{1}\left(F_{n}\right)$ since $[n(x), w(x)] \in U_{1}$. 
Thus, $\operatorname{ker} \theta$ is a normal subloop of the semicenter of $F_{n} / U_{1}\left(F_{n}\right)$. Since

$$
\left(F_{n} / U_{1}\left(F_{n}\right)\right) /\left(N\left(F_{n}\right) / U_{1}\left(F_{n}\right)\right) \cong F_{n} / N\left(F_{n}\right) \in L,
$$

the quotient loop is in the variety $L$. Therefore the free loop on $n$ generators in $L_{1}$ is in the class $C_{1}$.

Now, if $G$ is any loop in $L_{1}$, then there exists a free loop in $L_{1}$ with free generators $x_{1}, x_{2}, \ldots$ such that the mapping $\psi$ given by $x_{i} \rightarrow g_{i}$, for all $g_{i} \in G$, can be extended to a homomorphism $\psi$ of $F$ onto $G$. Let $N$ be a normal subloop of the semicenter of $F$ such that $F / N \in L$. Then $N \psi$ is a normal subloop of the semicenter of $G$. Define $\alpha: F / N \rightarrow G / N \psi$ by

$$
w\left(x_{1}, x_{2}, \ldots, x_{n}\right) N \alpha=w\left(g_{1}, g_{2}, \ldots, g_{n}\right) N \psi .
$$

$\alpha$ is clearly a homomorphism from $F / N$ onto $G / N \psi$ which implies that $G / N \psi \in L$. Therefore $L_{1} \subseteq C_{1}$.

To show that the converse is true, let $G$ be a loop with normal subloop $M$ in its semicenter and $G / M \in L$. Let $w_{1}=w_{2}$ be any identity in $L_{1}$. Then $w_{1}=w_{2}$ is equivalent to an identity $w\left(\left[n_{1}, u_{1}\right] \alpha_{1} \cdots\left[n_{t}, u_{t}\right] \alpha_{t}\right)=1$ where $n_{i}, u_{i}, i=1,2, \ldots, t$ are words in the free generators $x_{1}, x_{2}, \ldots, x_{n}$. Let $g_{1}, g_{2}, \ldots, g_{n} \in G$. Then for $i=1,2, \ldots, t$, $n_{i}\left(g_{1} M, g_{2} M, \ldots, g_{n} M\right)=M$ since $G / M \in L$. Therefore, $n_{i}\left(g_{1}, g_{2}, \ldots, g_{n}\right) \in M \subseteq$ semicenter of $G$. So, $\left[n_{i}(g), u_{i}(g)\right]=1$. Since, for any $\alpha \in I, 1 \alpha=1$, we have

$$
w\left(\left[n_{1}(g), u_{1}(g)\right] \alpha_{1}, \ldots,\left[n_{t}(g), u_{t}(g)\right] \alpha_{t}\right)=1 .
$$

It follows that $C_{1} \subseteq L_{1}$.

The proofs for the other cases are analogous to the one given.

THEOREM 7.3. If $L$ is closed under isotopy, then so are $L_{2}, L_{3}, L_{4}$ and $L_{5}$.

Proof. Let $G \in L_{2}$ with principal loop isotope $H$ given by $x \circ y=(x / a)(b \mid y)$. By Theorem 7.2, $G$ contains a normal subloop $N$ in its left nucleus with $G / N \in L$. By a result of Bruck, [6, p. 57, (v)], $G / N$ is isotopic to $H / b a N$. Since $L$ is closed under isotopy and $G / N \in L, H / b a N \in L$ also. By Bruck [6, p. 57, (iv)], the two left nuclei of the principal loop isotopes, $G$ and $H$, are isomorphic with the isomorphism given by $R_{b} R_{a}$. Therefore $N R_{b} R_{a}=(N b) a=b a N$ is normal in the left nucleus of $H$. Hence $H$ has a normal subloop $b a N$ in the left nucleus such that $H / b a N \in L$, which means that $H \in L_{2}$. Since every loop isotope of $G$ is isomorphic to a principal loop isotope of $G$, it follows that $L_{2}$ is closed under isotopy.

The proofs for $L_{3}, L_{4}$, and $L_{5}$ are similar.

If we call the loops in the classes $C_{2}-C_{5}$, left nuclear, middle nuclear, right nuclear, and central extensions by loops in $L$, then we can restate the results in Theorems 7.2 and 7.3 as

THEOREM 7.4. The classes of central, left nuclear, middle nuclear and right nuclear extensions by loops in an isotopically L-closed variety are isotopically L-closed varieties. 
8. Nilpotent varieties. If $G, H$, and $K$ are subsets of a loop $M$, then we define $(G, H)=\{[x, y] \alpha \mid x \in G, y \in H, \alpha \in I\}$ and

$$
(G, H, K)=\{[x, y, z] \alpha \mid x \in G, y \in H, z \in K, \alpha \in I\}
$$

where $I$ is the inner mapping group of $M$. We consider the following chain of subloops of a loop $G$.

$$
\begin{aligned}
G^{1} & =G, \\
G^{2} & =[G, G]=\langle t \mid t \in(G, G) \cup(G, G, G)\rangle, \\
G^{3} & =\left[G^{2}, G\right]=\left\langle t \mid t \in\left(G^{2}, G\right) \cup\left(G^{2}, G, G\right) \cup\left(G, G^{2}, G\right) \cup\left(G, G, G^{2}\right)\right\rangle, \\
\vdots & \\
G^{n+1} & =\left[G^{n}, G\right]=\left\langle t \mid t \in\left(G^{n}, G\right) \cup\left(G^{n}, G, G\right) \cup\left(G, G^{n}, G\right) \cup\left(G, G, G^{n}\right)\right\rangle,
\end{aligned}
$$

$G$ is nilpotent if for some nonnegative integer $n, G^{n+1}=1 ; G$ is nilpotent of class $n$ if $n$ is the least integer such that $G^{n+1}=1$.

The following theorem has a proof similar to the group case with the proper extension to the associators and the use of the inner mapping group to insure normality.

THEOREM 8.1. $G / Z(G)$ is nilpotent of class $n-1$ if and only if $G$ is nilpotent of class $n$.

Let $L_{n}$ be the class of all loops of nilpotency class $m \leqq n$.

THEOREM 8.2. $L_{n}$ is an isotopically $L$-closed variety. If $G$ has nilpotency class $n$, then so does every loop isotope of $G$.

Proof. The proof is by induction on $n$. For $n=1, L_{1}$ is the variety of abelian groups which is closed under isotopy and whose members all have nilpotency class one, disregarding the trivial group. Assume that the assertion holds for $n<k$. Let $V$ be the class of all central extensions by loops in $L_{k-1}$. By Theorem 8.1, if $G \in L_{k}$, then $G \in V$. Therefore $L_{k} \subseteq V$. Conversely, if $G \in V$, then $G$ has a normal subloop $N$ in its center and $G / N$ is in $L_{k-1}$. Therefore, there exists a homomorphism from $G / N$ onto $G / Z(G)$. By the induction hypothesis, $L_{k-1}$ is a variety. Therefore $G / Z(G) \in L_{k-1}$ and it follows that $V \subseteq L_{k}$. By Theorem 7.4, $V=L_{k}$ is an isotopically $L$-closed variety.

Now consider a loop $G$ of nilpotency class $k$ with principal loop isotope $H$. Then $G / Z(G)$ is isotopic to $H / Z(H)$. By Theorem $8.1, G / Z(G)$ is nilpotent of class $k-1$. By the induction hypothesis $H / Z(H)$ also has nilpotency class $k-1$, from which it follows that $H$ is nilpotent of class $k$. Since every loop isotope of $G$ is isomorphic to a principal loop isotope, every loop isotope of $G$ has nilpotency class $k$. 
T. Evans has given the set below of defining identities for $L_{2}$.

(i) $\left[\left[x_{1}, x_{2}\right], x_{3}\right]=1$;

(ii) $\left[\left[x_{1}, x_{2}\right], x_{3}, x_{4}\right]=1$;

(iii) $\left[\left[x_{1}, x_{2}, x_{3}\right], x_{4}, x_{5}\right]=1$;

(iv) $\left[\left[x_{1}, x_{2}, x_{3}\right], x_{4}\right]=1$;

(v) $\left[x_{1},\left[x_{2}, x_{3}\right], x_{4}\right]=1$;

(vi) $\left[x_{1},\left[x_{2}, x_{3}, x_{4}\right], x_{5}\right]=1$.

By Theorem 3.2, the variety of quasigroups $Q^{*}\left(L_{2}\right)$ is closed under isotopy and consists of all quasigroups, all of whose loop isotopes are nilpotent of class $n \leqq 2$. Since $L_{2}$ is isotopically $L$-closed, $Q^{*}\left(L_{2}\right)$ can be generated by taking all quasigroup isotopes of loops that have nilpotency class $n \leqq 2$. Since, the derived identities of a set of defining identities for $L_{2}$ is a basis for the identities of $Q^{*}\left(L_{2}\right)$, this quasigroup variety is defined by the identities given below where $[x, y, u, v]$ denotes the expression $\{[(x / u)(v \mid y)] /(v \mid[(y / u)(v \mid x)])\} \cdot u$ and $[x, t, z, u, v]$, the expression

$$
\{[([(x / u)(v \mid y)] / u)(v \mid z)] /(v \mid[(x / u)(v \mid[(y / u)(v \mid z)])])\} \cdot u .
$$

(i) $\left[\left[x_{1}, x_{2}, x_{3}, x_{4}\right], x_{5}, x_{3}, x_{4}\right]=x_{4} x_{3}$,

(ii) $\left[\left[x_{1}, x_{2}, x_{3}, x_{4}\right], x_{5}, x_{6}, x_{3}, x_{4}\right]=x_{4} x_{3}$,

(iii) $\left[\left[x_{1}, x_{2}, x_{3}, x_{4}, x_{5}\right], x_{6}, x_{7}, x_{4}, x_{5}\right]=x_{5} x_{4}$,

(iv) $\left[\left[x_{1}, x_{2}, x_{3}, x_{4}\right], x_{5}, x_{3}, x_{4}\right]=x_{4} x_{3}$,

(v) $\left[x_{1},\left[x_{2}, x_{3}, x_{4}, x_{5}\right], x_{6}, x_{4}, x_{5}\right]=x_{5} x_{4}$,

(vi) $\left[x_{1},\left[x_{2}, x_{3}, x_{4}, x_{5}, x_{6}\right], x_{7}, x_{5}, x_{6}\right]=x_{6} x_{5}$.

9. Varieties generated by classes of loops. It is well known that given any class of loops there is a loop variety whose identities are exactly the identities common to the members of the class. We impose a special condition on a class of loops in order to generate an isotopically $L$-closed variety.

THEOREM 9.1. If C is a class of loops containing all loop isotopes of the members of $C$, then the variety $L$ of loops generated by $C$ is closed under isotopy.

Proof. Let $G=\prod_{i \in I} G_{i}$ where $G_{i} \subseteq C$. Let $H$ be a principal loop isotope of $G$ given by $x \circ y=(x / a)(b \backslash y)$ where $a=\prod_{I} a_{i}, b=\prod_{I} b_{i}$. We show that $H=\prod_{I} H_{i}$, where $H_{i}$ is the isotope of $G_{i}$ given by $x_{i} \circ y_{i}=\left(x_{i} / a_{i}\right)\left(b_{i} \mid y_{i}\right), i \in I$. Let $\theta$ be the identity mapping from $H$ onto $\prod_{I} H_{i}$. If $x, y \in H$, then

$$
\begin{aligned}
(x \circ y) \theta & =(x / a)(b \mid y)=\left(\prod_{I} x_{i} / \prod_{I} a_{i}\right)\left(\prod_{I} b_{i} \backslash \prod_{I} y_{i}\right)=\prod_{I}\left(x_{i} / a_{i}\right) \prod_{I}\left(b_{i} \mid y_{i}\right) \\
& =\prod_{I}\left(\left(x_{i} / a_{i}\right)\left(b_{i} \mid y_{i}\right)\right)=\prod_{I}\left(x_{i} \circ y_{i}\right)=\prod_{I} x_{i} \circ \prod_{I} y_{i}=x \circ y=x \theta \circ y \theta .
\end{aligned}
$$

Hence $\theta$ is an isomorphism. Thus, a principal loop isotope of any cartesian product of members of $C$ is a cartesian product of members of $C$ and therefore belongs to $V(C)$, the variety generated by $C$. 
Let $A$ be a subloop of $G$ and $A^{\prime}$, a principal loop isotope of $A$ given by $x \circ y$ $=(x / c)(d \mid y)$ where $c, d \in A$. Then this isotopy induces an isotopy on $G$ given in the same way and applied to all elements of $G$. Let $H^{\prime}$ be the isotope of $G$ obtained in this way. Then $H^{\prime}$ is a principal isotope of $G$ and therefore belongs to $V(C)$ by the first part. Since $A^{\prime}$ is a subset of $H^{\prime}$ and is closed under the operations $\circ, \phi, \phi, A^{\prime}$ is a subloop of $H$. Therefore, $A^{\prime} \in V(C)$.

Now, let $B$ be a homomorphic image of $A$ and $B^{\prime}$, a principal loop isotope given by $x \circ y=(x \theta / n \theta)(m \theta \backslash y \theta)$ for some elements $m, n \in A$. Let $A^{\prime \prime}$ be the isotope of $A$ given by $x \circ y=(x / n)(m \mid y) . \theta$ is clearly a mapping from $A^{\prime \prime}$ onto $B^{\prime}$. Moreover, $(x \cdot y) \theta=[(x / n)(m \mid y)] \theta=(x \theta / n \theta)(m \theta \mid y \theta)=x \theta \circ y \theta$. Hence $\theta$ is a homomorphism from $A^{\prime \prime}$ onto $B^{\prime}$. Since $A^{\prime \prime} \in V(C)$ by the second part and $V(C)$ is closed under homomorphic images, $B^{\prime} \in V(C)$.

Let $G$ be any loop in $V(C)$ with loop isotope $K$. Then $K$ is isomorphic to some $G^{\prime}$, where $G^{\prime}$ is a principal loop isotope of $G . G$ is a homomorphic image of a subloop of a cartesian product of members of $C$. By what has already been shown, $G^{\prime} \in V(C)$. Since $K \cong G^{\prime}, K \in V(C)$. Therefore $V(C)$ is closed under isotopy.

Let $\circ$ stand for the operation on classes of loops of closure under isotopy. Thus, if $C$ is any class of loops, then $C \circ$ is the class of all loops isotopic to the loops in $C$. Since $C \circ \subseteq V(C)$, we have $V(C \circ \subseteq V((V(C)) \circ)$. Now, if $G$ is any loop in $V(C)$, then $G \in V(C \circ)$ which is closed under isotopy by Theorem 9.1. Hence, if $H$ is a loop isotope of $G$, then $H \in V(C \circ)$ which implies that $(V(C)) \circ \subseteq(C \circ)$. Therefore, $V((V(C)) \circ \subseteq V(V(C \circ)) \subseteq V(C \circ)$. Thus, we have the following result.

COROLlaRY 9.2. If $C$ is any class of loops, then $V(C \circ)=V((V(C)) \circ)$.

COROLlARY 9.3. If there exists a finite anti-associative loop with every loop isotope anti-associative, then there is an anti-associative isotopically L-closed variety.

Proof. If $G_{1}$ is a finite anti-associative loop, then $G$ has only a finite number of nonisomorphic loop isotopes, $G_{2}, G_{3}, \ldots, G_{n}$. Evans in [9] has shown that every anti-associative loop satisfies an identity of the form $x p(x)=1$, where $p(x)$ is a word in one variable in the subloop $[F, F]$ of the free loop $F$. Let $x p_{i}(x)=1$ be an identity satisfied by $G_{i}, i=1,2, \ldots, n$. Then $G_{1} \times G_{2}$ satisfies the identity $\left(x p_{2}(x)\right)$ $\cdot\left[p_{1}\left(x \cdot p_{2}(x)\right)\right]=1$ which is again an anti-associative identity. Continuing in this way we can find an anti-associative identity $x p(x)=1$ which is satisfied by $G_{1} \times G_{2}$ $\times \cdots \times G_{n}$. Therefore, the variety $V$ generated by $G$ and its isotopes is antiassociative. By Theorem $9.1, V$ is isotopically $L$-closed.

\section{REFERENCES}

1. J. Aczél, Quasigroups, nets and nomograms, Advances in Math. 1 (1965), 383-450. MR 33 \#1395.

2. J. Aczél, V. Belousov and M. Hosszú, Generalized associativity and bisymmetry on quasigroups, Acta Math. Acad. Sci. Hungar. 11 (1960), 127-136. MR 26 \#4018.

3. A. Albert, Quasigroups. I, Trans. Amer. Math. Soc. 54 (1943), 507-519. MR 5, 229. 
4. V. Belousov, Balanced identities in quasigroups, Mat. Sb. 70 (112) (1966), 55-97. (Russian) MR 34 \#2757.

5. V. Belousov and V. Ryžkov, On a method of obtaining closure figures, Mat. Issled. 1 (1966), 140-150. (Russian) MR 36 \#6526.

6. R. Bruck, $A$ survey of binary systems, Ergebnisse der Mathematik und ihrer Grenzgebiete, Heft 20, Reihe: Gruppentheorie, Springer-Verlag, Berlin and New York, 1958. MR 20 \#76.

7. - Some results in the theory of quasigroups, Trans. Amer. Math. Soc. 55 (1944), 19-52. MR 5, 229.

8. T. Evans, A note on the associative law, J. London Math. Soc. 25 (1950), 196-201. MR $12,75$.

9. - Identical relations in loops. I, Austral. J. Math. (to appear).

10. - Identical relations in loops. II (in preparation).

11. - On multiplicative systems defined by generators and relations. I: Normal form theorems, Proc. Cambridge Philos. Soc. 47 (1951), 637-649. MR 13, 312.

12. J. Osborn, Loops with the weak inverse property, Pacific J. Math. 10 (1960), 295-304. MR 22 \#2660.

13. A. Sade, Demosian systems of quasigroups, Amer. Math. Monthly 68 (1961), 329-337. MR 25 \#2020.

EMORY UNIVERSITY,

Atlanta, Grorgia 30322

Spelman College,

Atlanta, Georgia 30314 